\title{
Visible photoluminescence of silicon-based nanostructures: Porous silicon and small silicon-based clusters
}

\author{
Yoshihiko Kanemitsu, Katsunori Suzuki, Hiroshi Uto, and Yasuaki Masumoto \\ Institute of Physics, University of Tsukuba, Tsukuba, Ibaraki 305, Japan \\ Takahiro Matsumoto \\ Electronics Research Laboratories, Nippon Sieel Corporation, Sagamihara, Kanagawa 229, Japan \\ Soichiro Kyushin, Koichi Higuchi, and Hideyuki Matsumoto \\ Department of Chemistry, Gunma University, Kiryu, Gunma 376, Jupan
}

(Received 10 June 1992; accepted for publication 16 September 1992)

\begin{abstract}
We have studied and compared the optical properties of both porous $\mathrm{Si}$ and the chemically synthesized planar and cubic Si skeleton clusters. Broad photoluminescence with large Stokes shifts were observed at the visible region in both samples. Spectroscopic analysis suggests that the surface of porous $\mathrm{Si}$ is similar to a condensation of $\mathrm{Si}$ clusters. Small Si clusters play a key role in the strong room-temperature photoluminescence in porous Si.
\end{abstract}

Optical and electronic properties of nanometer-size semiconductor crystallites have attracted much attention, because they exhibit new quantum phenomena and have potential for becoming novel and future photonic devices. ${ }^{1,2}$ Very recently, a great deal of research effort is focused on low-dimensional nanostructures made from indirect-gap semiconductors such as $\mathrm{Si}^{3-5}$ or $\mathrm{Ge}^{6}$ Especially, the discovery of the strong luminescence from $\mathrm{Si}$ nanostructures fabricated by electrochemical anodization, often called porous $\mathrm{Si}$, is an extremely important scientific breakthrough with enormous technological implications, since it opens up new possibilities for $\mathrm{Si}$ as a material for optoelectronic applications.

The origin and mechanism of strong visible luminescence in porous $\mathrm{Si}$ are currently under discussion ${ }^{7}$ and the possibility of quantum confinement effects in $\mathrm{Si}$ wires or dots is suggested by the following observations; (a) the blue shift of the photoluminescence (PL) spectrum of porous $\mathrm{Si}$ was observed when the Si column size was reduced by increasing the etching time in HF solutions, ${ }^{5,8}$ (b) the temperature dependence of the PL intensity ${ }^{9}$ suggests the enhancement of the exciton binding energy, and (c) Raman studies ${ }^{10,11}$ show that porous Si maintains the crystalline character of $\mathrm{Si}$ and typical crystalline size of about 3 $\mathrm{nm}$.

On the other hand, the importance of the localized states at the surface of nanostructures was pointed out by several authors. ${ }^{7}$ With a large surface-to-volume ratio in the highly porous structure, the influence of surface effect on luminescence processes is enhanced and the surface of porous $\mathrm{Si}$ is responsible for the origin of luminescence. The studies of luminescence degradation in various ambient gases ${ }^{12}$ and $x$-ray photoelectron spcctroscopy ${ }^{13}$ suggest that the electronic properties at the surface in porous Si play a key role in the strong luminescence. Moreover, silicon based compounds, especially, siloxene $\left(\mathrm{Si}_{6} \mathrm{O}_{3} \mathrm{H}_{6}\right)$ derivates are also proposed as an origin of the strong luminescence of porous $\mathrm{Si}^{14}$ If the surface of $\mathrm{Si}$ crystallites is terminated by hydrogen atoms, siloxene, or other silicon compounds, the electronic properties of the near-surface region are different from the inside of Si crystallites. In this case, it is natural to consider that the surface is formed with a new nanometersize material.

In this lelter, we have studied and compared the optical properties of both porous $\mathrm{Si}$ and the chemically synthesized Si skeleton clusters. PL and absorption spectra and picosecond PL decay measurements show that optical properties of porous $\mathrm{Si}$ are similar to those of planar and cubic $\mathrm{Si}$ skeleton clusters. The rough surface of porous $\mathrm{Si}$ is regarded as a condensation of small $\mathrm{Si}$ clusters and the clusterlike surface region plays a key role in the strong visible luminescence.

Porous silicon was prepared as follows. The substrates were (100)-oriented $3.5-4.5 \Omega \mathrm{cm}$ resistivity $p$-type silicon. Thin Al films wcrc evaporated on the back of the wafers to form a good ohmic contact. The anodization was carried out in $\mathrm{HF}$-ethanol solution ( $\mathrm{HF}: \mathrm{H}_{2} \mathrm{O}: \mathrm{C}_{2} \mathrm{H}_{5} \mathrm{OH}=1: 1: 2$ ) at a constant current density of $10 \mathrm{~mA} / \mathrm{cm}^{2}$ for $5 \mathrm{~min}$. Furthermore, photochemical etching of the wafer was carried out for 2 min in HF-ethanol solution under illumination with a $500 \mathrm{~W}$ tungsten lamp for a distance of $20 \mathrm{~cm}^{15}$ After the photochemical etching, the wafers were rinsed in deionized water for $2 \mathrm{~min}$. These etching treatments cause an increase of the PL intensity and a blueshift of the PL peak. Figure $1(\bar{a})$ shows a typical image of a porous $\mathrm{Si}$ sample by transmission electron microscope (TEM) analysis. The local structure of the porous silicon is a network of interconnected crystalline silicon islands. The shape of the islands is spherelike rather than wirelike. The size of Si crystallite islands ranges from several $\mathrm{nm}$ to $20 \mathrm{~nm}$. Our TEM observations are similar to the report by Cullis and Canham. ${ }^{8}$

A planar Si skeleton cluster (syn-tricyclooctasilane) and cubic Si skeleton cluster (octasilacubane) were synthesized and used as model Si-based materials consisting of a small number of $\mathrm{Si}$ atoms. The structures are illustrated in Figs. 1(b) and 1(c). Synthetic and purification methods were described in Ref.16.

The PL spectra of porous $\mathrm{Si}$ and Si skeleton clusters were measured in a vacuum by using $325 \mathrm{~nm}$ excitation 

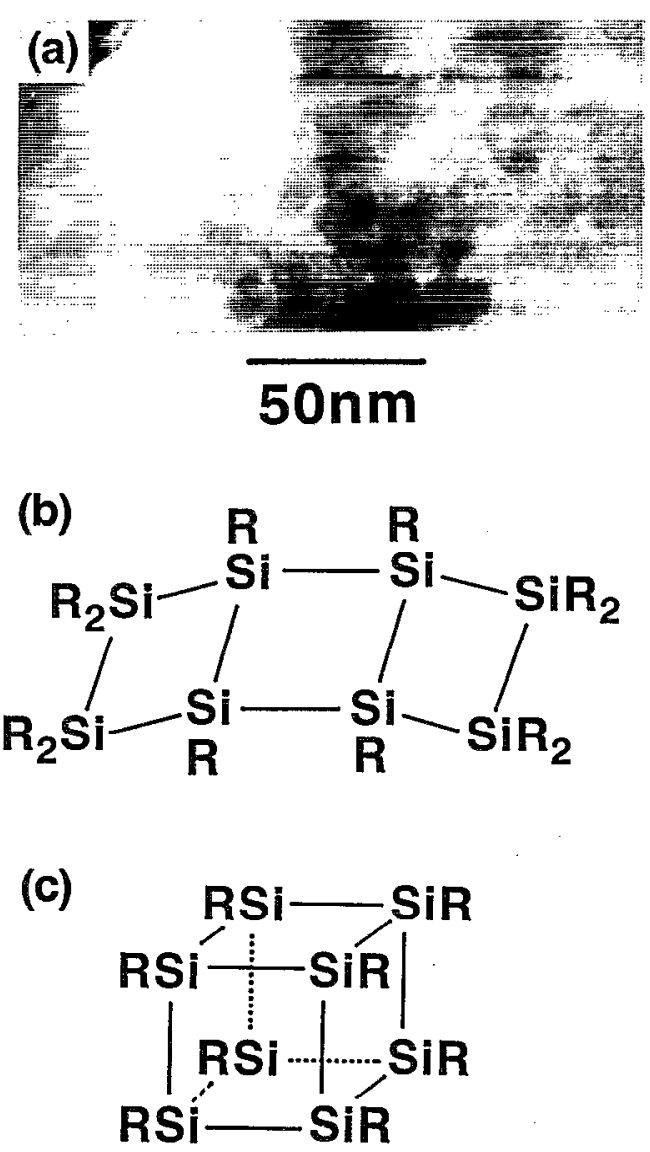

FIG. 1. (a) TEM image of porous $\mathrm{Si}$, (b) a planar Si skeleton cluster, syn-tricyclooctasilane ( $R=$ isopropyl), and (c) a cubic Si skeleton cluster, octasilacubane ( $R=t$-butyldimethylsilyl).

light from a He-Cd laser. Excitation spectra of the PL at the peak photon energy were measured by using a Xe-lamp and a monochromator. Picosecond PL decay under a 1 ps and $300 \mathrm{~nm}$ laser excitation was measured using a monochromator of subtractive dispersion and a synchroscan streak camera. The calibration of the spectral sensitivity of the whole measuring system was performed by using a tungsten standard lamp. Spectroscopic data were measured at room temperature.

Figure 2(a) shows the excitation and PL spectra in porous Si. A gradual rise in the excitation spectrum is observed at photon energies above $2 \mathrm{eV}$. An excitation edge exists near $\sim 3.2 \mathrm{eV}$, and broad PL spectrum is observed at the peak value of $1.98 \mathrm{eV}$. There exists a large Stokes shift of $\sim 1 \mathrm{eV}$ between the PL peak and the edge of excitation spectrum. Ren and Dow ${ }^{17}$ theoretically calculated the size dependence of the band gap of Si crystallites with hydrogenated surfaces. According to their calculations, the band gap of crystallites of $3 \mathrm{~nm}$ diam which is a characteristic size of porous $\mathrm{Si}^{8,10,11}$ is $\sim 1.5 \mathrm{eV}$. The observed edge energy of $\sim 3 \mathrm{eV}$ and PL peak of $\sim 2 \mathrm{eV}$ are larger than the theoretical band gap $(\sim 1.5 \mathrm{eV})$ of $3 \mathrm{~nm}$ crystallites. These imply that a region containing a small number of $\mathrm{Si}$ atoms (e.g., clusterlike surface region in $\mathrm{Si}$ crystallites) exhibits the strong luminescence.

Figures 2(b) and 2(c) show the absorption and PL

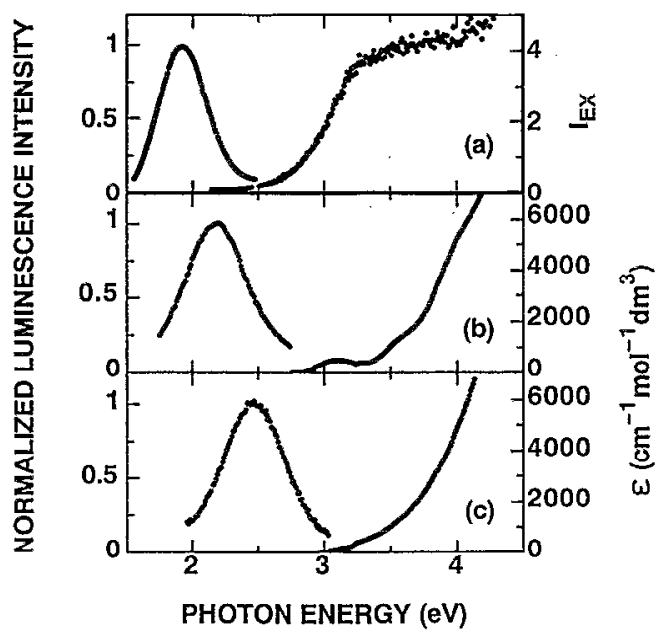

FIG. 2. (a) Excitation ( $I_{\mathrm{EX}}$ ) and PL spectra in porous silicon. Molar extinction coefficients $\epsilon$ and PL spectra in a planar Si skeleton cluster (b) and in a cubic Si skeleton cluster (c).

spectra in planar and cubic Si skeleton clusters, respectively. The absorption spectra of Si skeleton clusters solved in tetrahydrofuran (THF) were measured and the molar extinction coefficients are plotted in this figure. In a planar Si cluster, the lowest excitation state is observed at $3.09 \mathrm{eV}$ and a broad PL spectrum is observed with the peak of 2.25 $\mathrm{eV}$, thus, the Stokes shift is $0.84 \mathrm{eV}$. In a cubic Si cluster, the absorption edge is observed at $\sim 3.2 \mathrm{eV}$ and a broad PI, spectrum is also observed with the peak of $2.50 \mathrm{eV}$. Both the broad PL spectra and the large Stokes shifts in planar and cubic Si clusters are different from those in onedimensional chainlike Si clusters with a direct gap nature. ${ }^{18}$ We note that even in very small Si clusters the shape of the cluster plays an important role in determining optical properties. The effect of dimensionality on electronic structures in small $\mathrm{Si}$ skeleton clusters is discussed elsewherc. ${ }^{19}$ Although we were not able to obtain the absolute PL quantum efficiencies of both porous $\mathrm{Si}$ and Si clusters, we estimated that there is no significant difference of the relative $\mathrm{PL}$ quantum efficiency between porous $\mathrm{Si}$ and $\mathrm{Si}$ clusters. The characteristics of PL and excitation spectra of porous $\mathrm{Si}$ are similar to those of $\mathrm{Si}$ clusters.

Figure 3 shows the picosecond PL decay at peak energies in porous Si and planar and cubic Si skeleton clusters. The picosecond decay is clearly observed in porous $\mathrm{Si}$. Many researchers ${ }^{7}$ reported that the PL decay shows a nonexponential behavior with typical time constants ranging from nanoseconds to milliseconds. This behavior is primarily caused as a consequence of the inhomogeneous size distribution of Si nanostructures as shown in Fig. 1. The PL decay exhibits nonexponential behavior even in picosecond time scale. On the other hand, in planar and cubic clusters fast PL decay is also observed in picosecond timescale. The rate of radiative recombination determined by the intrinsic lifetime of excited states is reflected by the initial fast component of the PL decay in an inhomogeneous system such as porous $\mathrm{Si}$, since the long PL decay components are caused by spatial tunneling, thermally ac- 


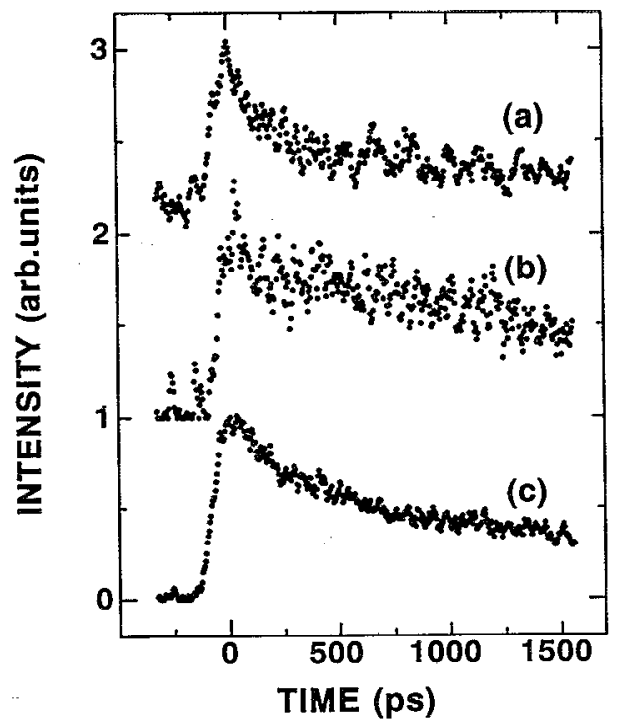

FIG. 3. Picosecond PL decay at the peak photon energies; (a) porous $\mathrm{Si}$, (b) a planar Si skeleton cluster, and (c) a cubic Si skeleton cluster.

tivated carrier diffusion, and so on. ${ }^{20}$ Therefore, the essential agreement of the picosecond PL decay between porous $\mathrm{Si}$ and $\mathrm{Si}$ clusters implies that the luminescent properties of porous $\mathrm{Si}$ are similar to those of small planar and cubic clusters.

From our experimental findings of the quantitative agreement of optical properties between porous $\mathrm{Si}$ and $\mathrm{Si}$ clusters, it is concluded that the substance causing the luminescence in porous Si shows similar optical characteristics to the Si-based clusters. The rough surface of porous $\mathrm{Si}$ is considered as a condensation of Si skeleton clusters. The near-surface region consisting of small clusters plays an essential role in determining the strong luminescence properties. Moreover, Brandt et al. ${ }^{14}$ pointed out that the electronic properties of siloxene compounds with sixfold $\mathrm{Si}$ rings are similar to those of porous $\mathrm{Si}$ and that small $\mathrm{Si}$ skeleton clusters also play an essential role in the luminescence process of these siloxene compounds. The notion of a small Si skeleton cluster gives a better understanding of the luminescent mechanism of porous Si. At present, it is considered that the near-surface region consists of small $\mathrm{Si}$ clusters and the clusterlike near-surface region contributes the strong room-temperature luminescence.

In conclusion, we have studied and compared the optical properties of porous silicon and chemically synthesized Si skeleton clusters. Optical properties of planar and cubic Si clusters are similar to those of porous silicon. Spectroscopic analysis suggests that the near-surface region of porous $\mathrm{Si}$ consists of small $\mathrm{Si}$ clusters and that this origin plays a key role in the strong room-temperature photoluminescence.

The author would like to thank Dr. F. Sakuma of National Research Laboratory of Metrology for the kind loan of a standard lamp.

${ }^{1}$ See, for example, L. Brus, IEEE J. Quantum Electron. QE-22, 1909 (1986); L. Brus, Appl. Phys. A 53, 465 (1991); and references therein.

${ }^{2}$ See, for example, E. Hanamura, Phys. Rev. B 38, 1288 (1988); Y. Kayanuma, Phys. Rev. B 38, 9772 (1988); and references therein.

${ }^{3}$ S. Furukawa and T. Miyasato, Phys. Rev. B 38, 5726 (1988).

${ }^{4}$ V. Lehmann and U. Gosele, Appl. Phys. Lett. 58, 856 (1991).

${ }^{5}$ L. T. Canham, Appl. Phys. Lett. 57, 1046 (1990).

${ }^{6}$ Y. Kanemitsu, H. Uto, Y. Masumoto,and Y. Maeda, Appl. Phys. Lett. 61, 2187 (1992); Y. Maeda, N. Tsukamoto, Y. Yazawa, Y. Kanemitsu, and Y. Masumoto, Appl. Phys. Lett. 59, 3168 (1991).

${ }^{7}$ See, for example, Abstract Book of Fall Meeting of the Matcrials Rcsearch Society, Symposium AA (Materials Research Society, Boston, 1991).

${ }^{8}$ A. G. Cullis and L. T. Canham, Nature 353, 335 (1991).

${ }^{9}$ S. Gardelis, J. S. Rimmer, P. Dawson, B. Hamilton, R. A. Kubiak, T. E. Whall, and E. H. Parker, Appl. Phys. Lett. 59, 2118 (1991).

${ }^{10}$ R. Tsu, H. Shen, and M. Dutta, Appl. Phys. Lett. 60, I12 (1992).

"Z. Sui, P. P. Leong, I. P. Herman, G. S. Higashi, and H. Temkin, Appl. Phys. Lett. 60, 2086 (1992).

${ }^{12}$ M. A. Tischler, R. T. Collins, J. H. Stathis, and J. C. Tsang, Appl. Phys. Lett. 60, 639 (1992).

${ }^{13}$ R. P. Vasquez, R. W. Fathauer, T. George, A. Ksendzov, and T. L. Lin, Appl. Phys. Lett. 60, 1004 (1992).

${ }^{14}$ M. S. Brandt, H. D. Fuchs, M. Stutzmann, J. Weber, and M. Cardona, Solid State Commun. 81, 307 (1992).

${ }^{15}$ N. Koshida and H. Koyama, Jpn. J. Appl. Phys. 30, L1221 (1990).

${ }^{16} \mathrm{H}$. Matsumoto, H. Miyamoto, N. Kojima, and Y. Nagai, J. Chem. Soc. Chem. Commun. 1316 (1987); H. Matsumoto, K. Higuchi, Y. Hoshino, H. Koike, Y. Naoi, and Y. Nagai, J. Chem. Soc. Chem. Commun. 1083 (1988).

${ }^{17}$ S. Y. Ren and J. D. Dow, Phys. Rev. B 45, 6492 (1992).

${ }^{18}$ Y. Kanemitsu, K. Suzuki, Y. Nakayoshi, and Y. Masumoto, Phys. Rev. B 46, 3916 (1992).

${ }^{19}$ Y. Kanemitsu, K. Suzuki, Y. Masumoto, K. Sato, S.Kyuushin, and H. Matsumoto (unpublished).

${ }^{20} \mathrm{R}$. A. Street, in Semiconductors and Semimetals, edited by J. I. Pankove (Academic, Orlando, 1984), Vol. 21, Part. B, p. 197. 